\title{
Natural bioceramics: our experience with changing perspectives in the reconstruction of maxillofacial skeleton
}

\author{
Vivekanand Sabanna Kattimani, Krishna Prasad Lingamaneni \\ Department of Oral and Maxillofacial Surgery, Sibar Institute of Dental Sciences, Guntur, India
}

\begin{abstract}
J Korean Assoc Oral Maxillofac Surg 2019;45:34-42)
Objectives: Various bone graft substitute materials are used to enhance bone regeneration in the maxillofacial skeleton. In the recent past, synthetic graft materials have been produced using various synthetic and natural calcium precursors. Very recently, eggshell-derived hydroxyapatite (EHA) has been evaluated as a synthetic bone graft substitute. To assess bone regeneration using EHA in cystic and/or apicectomy defects of the jaws through clinical and radiographic evaluations.

Materials and Methods: A total of 20 patients were enrolled in the study protocol (CTRI/2014/12/005340) and were followed up at 4, 8, 12, and 24 weeks to assess the amount of osseous fill through digital radiographs/cone-beam computed tomography along with clinical parameters and complications. Wilcoxon matched pairs test, means, percentages and standard deviations were used for the statistical analysis.

Results: The sizes of the lesions in the study ranged from 1 to $4 \mathrm{~cm}$ and involved one to four teeth. The study showed significant changes in the formation of bone, the merging of material and the surgical site margins from the first week to the first month in all patients (age range, 15-50 years) irrespective of the size of the lesions and the number of teeth involved. Bone formation was statistically significant from the fourth to the eighth week, and the trabecular pattern was observed by the end of 12 weeks with uneventful wound healing.

Conclusion: EHA showed enhancement of bone regeneration, and healing was complete by the end of 12 weeks with a trabecular pattern in all patients irrespective of the size of the lesion involved. The study showed enhancement of bone regeneration in the early bone formative stage within 12 weeks after grafting. EHA is cost effective and production is environment friendly with no disease transfer risks. Thus, natural bioceramics will play an important role in the reduction of costs involved in grafting and reconstruction.
\end{abstract}

Key words: Apicoectomy, Wound healing, Grafts, Osteoconduction

[paper submitted 2018. 1. 2 / revised 1st 2018. 4. 10, 2nd 2018. 4. 22, 3rd 2018. 4. 27 / accepted 2018. 5. 6]

\section{Introduction}

Biomaterials are used as biocompatible scaffold systems, which allow the migration, proliferation, and differentiation of either resident or externally delivered cells to promote new bone formation ${ }^{1-4}$. A wide variety of biomaterials have been used for craniofacial bone augmentation ${ }^{1,3,4}$. These can be divided into organic and inorganic materials, where calcium phosphate bioceramics represent most inorganic scaffolds ${ }^{1,3}$.

\section{Vivekanand Sabanna Kattimani}

Department of Oral and Maxillofacial Surgery, Sibar Institute of Dental Sciences, Takkellapadu, Guntur 522509, India

TEL: +91-9492473628 FAX: +91-863-2292139

E-mail:drvivekanandsk@gmail.com

ORCID: https://orcid.org/0000-0002-9812-7207

(c) This is an open-access article distributed under the terms of the Creative Commons Attribution Non-Commercial License (http://creativecommons.org/ licenses/by-nc/4.0/), which permits unrestricted non-commercial use, distribution, and reproduction in any medium, provided the original work is properly cited.

Copyright (C) 2019 The Korean Association of Oral and Maxillofacial Surgeons. All rights reserved.
The basic rationale behind such materials use is an attempt to mimic an inorganic composition of native bone (hydroxyapatite [HA], a natural bioceramic) to form a strong and durable natural biomaterial for early mineralization ${ }^{1,3}$.

Bioceramics is an advancing front in the reconstruction of maxillofacial skeleton defects. Advancing technology for the production of synthetic graft materials has led surgeons to quest for artificial bone regeneration ${ }^{1-3}$. Various bone graft substitute materials are used to enhance bone regeneration ${ }^{1,2}$. In the recent past, eggshell-derived hydroxyapatite (EHA) has been evaluated as a synthetic bone graft substitute and has changed the face of regenerative science ${ }^{5,6}$. Thus, this study was planned to assess bone regeneration using HA synthesized from a natural calcium precursor derived from chicken eggshells for the grafting of cystic and/or apicectomy defects in the mandible and maxilla using digital radiographs/ cone-beam computed tomography $(\mathrm{CBCT})$. 


\section{Materials and Methods}

\section{Study design and materials}

Our study was performed in the Department of Oral and Maxillofacial Surgery and the Department of Conservative and Endodontics of Sibar Institute of Dental Sciences (Guntur, India) from the year 2015 through 2017. A total of twentyone patients (14 males and seven females; age range, 15-50 years) requiring grafting after cystectomy and/or apicectomy were enrolled. However, only 20 patients were assessed in the study, because one patient was excluded from the assessment. The study protocol was approved by the Institutional Ethics Committee of Sibar Institute of Dental Sciences (IEC 16/09/2014) and prospectively registered with the Clinical Trial Registry of India - CTRI/2014/12/005340.

1) Inclusion and exclusion criteria for patient selection

- Patients with moderately sized (more than $1 \mathrm{~cm}$ for the largest diameter) periapical cystic lesions of the maxilla and mandible involving one or more teeth, as confirmed by clinical and radiological evaluations, were included in the study.

- Patients who were readily available and willing to return for periodic recalls and reviews were assessed.

- All cases were screened for any systemic diseases such as diabetes, and hypertension, and medically compromised patients were excluded in the evaluation process.

- Patients with the gross mobility of involved teeth due to moderate bone loss or frankly infected cysts were excluded from the study.

- Vulnerable groups and special category people were excluded from the evaluation according to an Indian council for medical research guidelines.

2) Graft material used in the study

The EHA used in this study was synthesized from a cal- cium precursor from chicken eggshells ${ }^{7}$. We prepared the HA using the microwave process published in a previous study ${ }^{7,8}$. In this study, we used nanocrystalline eggshell-derived calcium-deficient HA measuring an average of $78 \mathrm{~nm}$ with a typical flower-like structure, which contained the $\mathrm{Ca}, \mathrm{P}$, and $\mathrm{Mg}$ trace elemental composition needed for bone formation ${ }^{7}$.

\section{Surgical protocol}

All patients were assessed preoperatively for the extent of their lesions using radiographs/CBCT, and if necessary, surgical stents were prepared for through and through (tunnel) lesions for palatal soft tissue support post-surgically. The patients and attenders received explanations about the study protocol and written consent was obtained. All patients underwent root canal treatments. Each apicectomy or cystectomy was performed under local anesthesia with all aseptic precautions within three days of obturation. A full-thickness mucoperiosteal trapezoidal or quadrangular flap was elevated. The cystic lining was enucleated, and the lesion was curettaged. Apicectomy was performed by cutting 2 to $3 \mathrm{~mm}$ of root end, and the retrograde filling was done using a glass ionomer cement/mineral trioxide aggregate.(Fig. 1. A) The defects were grafted with EHA particles, and care was taken so that no graft particles were placed outside the cavity under the mucoperiosteal flap.(Fig. 1. B) Wound closure was done using 3-0 Mersilk suture.(Fig. 1. C) In all cases, antibiotics and analgesics were prescribed for five days. Patients were recalled for suture removal seven days postsurgery.

\section{Clinical and radiographic assessments}

The patients were followed up over a period of 24 weeks post-surgically (Fig. 1. D) at intervals of 4, 8, and 12 weeks to assess the amount of osseous fill using digital radiographs (Fig. 2)/CBCT.(Fig. 3) Clinically, the patients were assessed
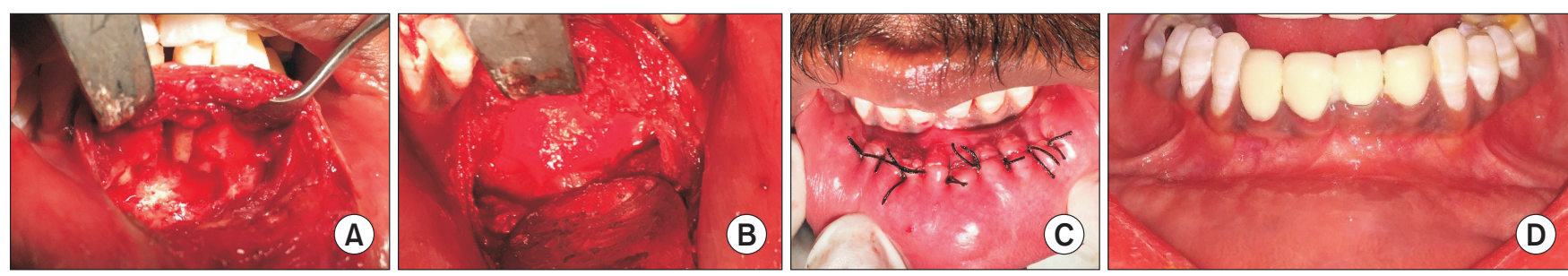

Fig. 1. A. Bony defect and loss of labial cortical plate. B. Bony defect filled with eggshell-derived hydroxyapatite. C. Wound closure. D. Well-healed wound with permanent crowns in place.

Vivekanand Sabanna Kattimani et al: Natural bioceramics: our experience with changing perspectives in the reconstruction of maxillofacial skeleton. J Korean Assoc Oral Maxillofac Surg 2019 

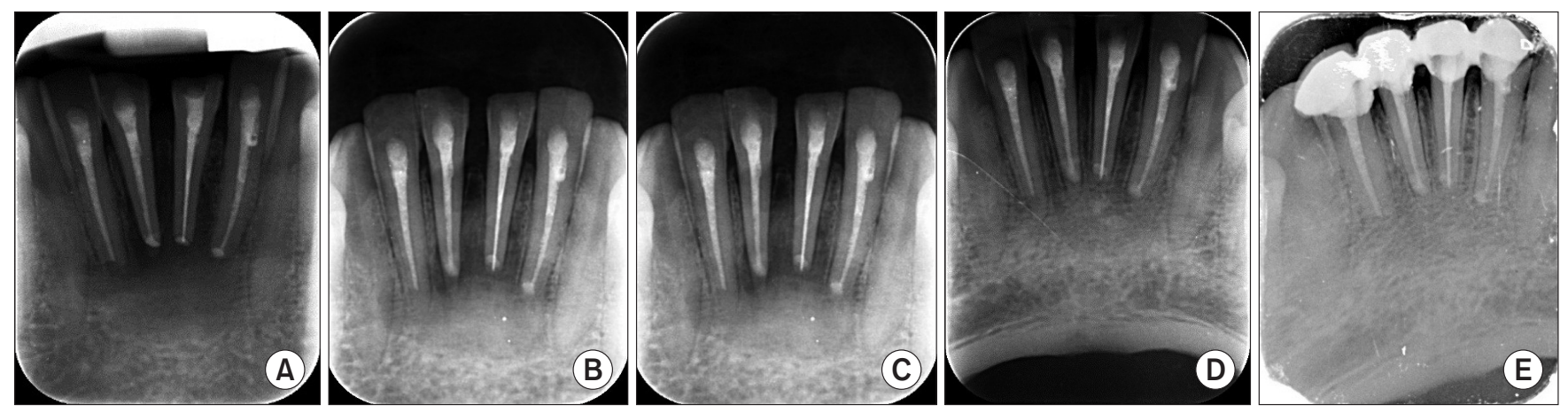

Fig. 2. Intraoral periapical radiographs showing radiological evaluations of lesion. A. Immediately after grafting. B. Four weeks postoperative. C. Eight weeks postoperative. D. Twelve weeks postoperative. E. Twenty-four weeks postoperative.

Vivekanand Sabanna Kattimani et al: Natural bioceramics: our experience with changing perspectives in the reconstruction of maxillofacial skeleton. J Korean Assoc Oral Maxillofac Surg 2019

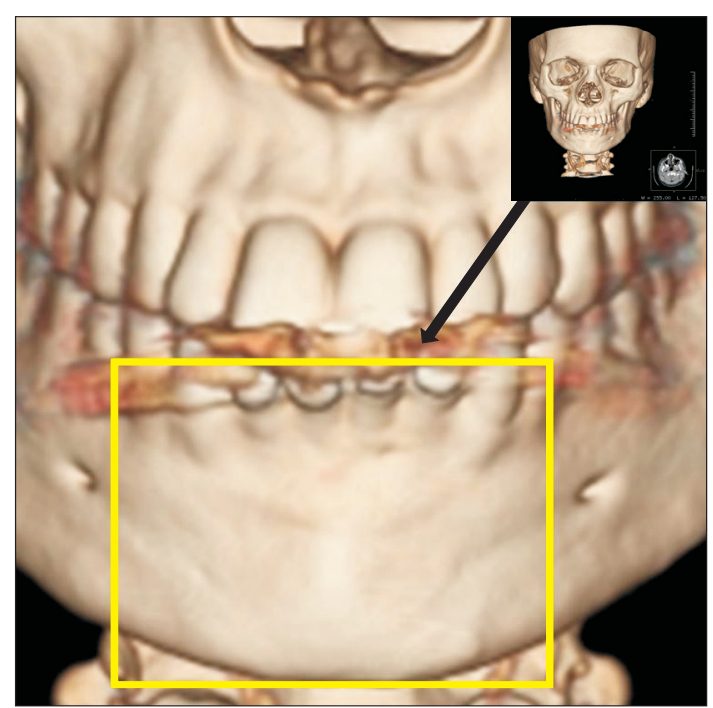

Fig. 3. Three-dimensional computed tomography of facial bone (frontal view) showing the complete bone formation after 24 weeks postoperative.

Vivekanand Sabanna Kattimani et al: Natural bioceramics: our experience with changing perspectives in the reconstruction of maxillofacial skeleton. J Korean Assoc Oral Maxillofac Surg 2019

for wound healing/dehiscence, infection, persistent pain, sinus, or fistulas. Radiographically, the surgical site margin (Fig. 4. A), bone formation characteristics (Fig. 4. B), radicular healing patterns, and density of bone formation were evaluated $^{9,10}$. The radiographs were assessed by two endodontists who were blinded for the time duration of the follow-up and requested to fill out the assessment sheets. If a discrepancy existed, a third examiner (oral radiologist) was requested to assess the radiograph.

\section{Statistical analysis}

The results were tabulated using Microsoft Excel 2010
(Microsoft, Redmond, WA, USA). The means, percentages, and Wilcoxon matched pairs test were used for statistical analysis using IBM SPSS Statistics 20.0 (IBM, Armonk, NY, USA). Kappa correlation was considered to assess the degree of observer agreement for radiological assessment.

\section{Results}

\section{Clinical observations}

All patients (Table 1) showed well-healed wound, except in one female patient wound dehiscence was seen, who was excluded from the study protocol. The sutures were removed after seven days without any infections, persistent pain, sinus, or fistula irrespective of the sizes of the lesions (Table 1), which were measured intraoperatively.

\section{Radiological observations}

Significant changes were observed in the merging of the material and surgical site outline in all of the patients over a period of eight weeks; $95 \%$ of cases showed the absence of demarcation between material and the surgical outline and this process was almost complete by the end of 12 weeks. (Table 2) The comparison of the merging of material and surgical site outline was significant between the first week and the fourth, eighth, twelfth, and twenty-fourth weeks with significant $(P<0.05) P$-values irrespective of the size of the lesion, the site, or the number of teeth involved.(Table 3) Bone formation was $90 \%$ specular and $10 \%$ trabecular in the grafted sites by the end of eight weeks (Table 4) with a significant $P$-value (Table 5), and $90 \%$ trabecular pattern was seen by the end of 12 weeks. The radiological evaluation of 
A

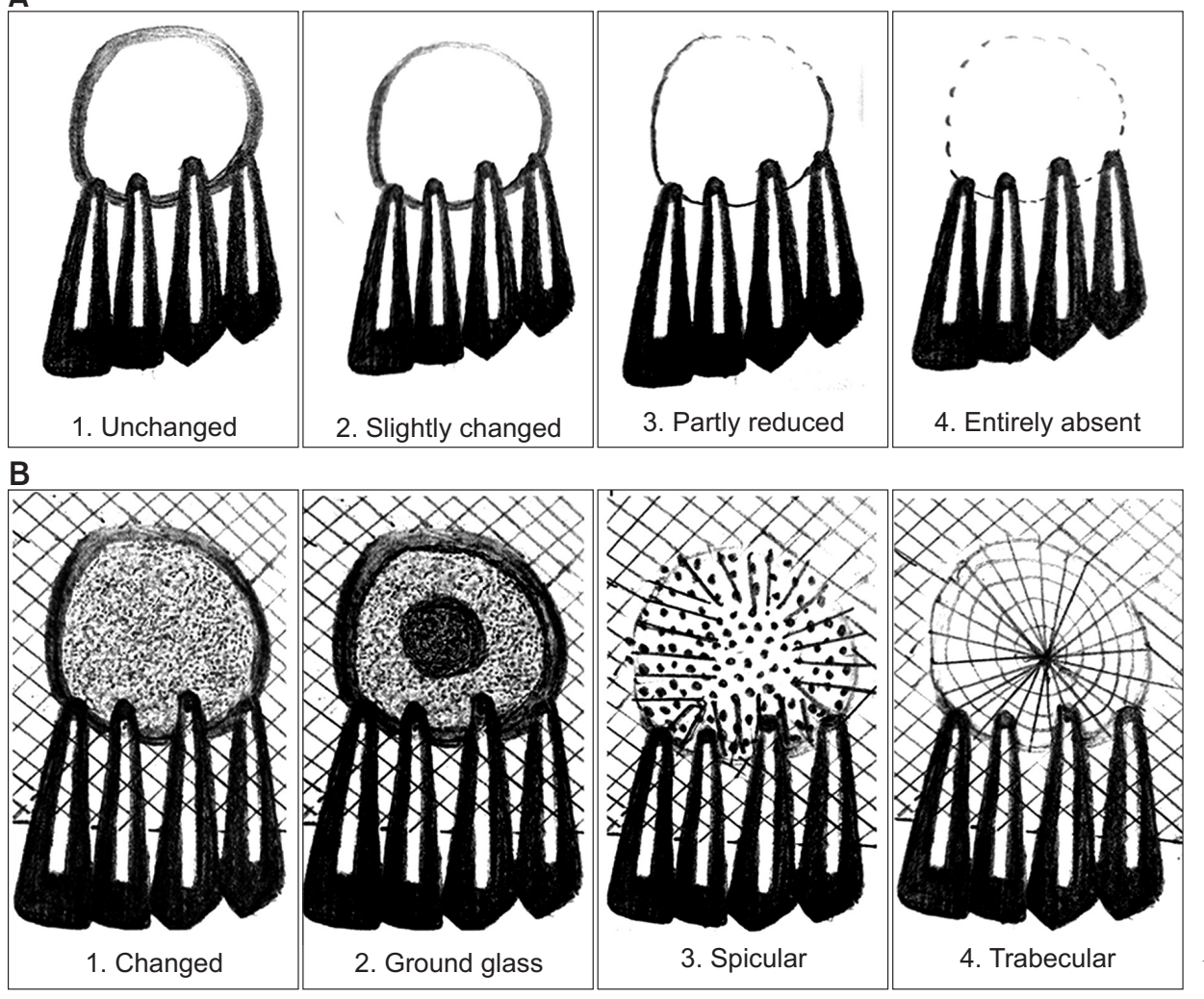

Fig. 4. Illustration showing the radiological evaluation of surgical site outline (A) and bone formation characteristics (B). Reproduced from the article of Kattimani et al. (Indian J Dent Res 2014;25:594-601) $)^{10}$ with original copyright holder's permission.

Vivekanand Sabanna Kattimani et al: Natural bioceramics: our experience with changing perspectives in the reconstruction of maxillofacial skeleton. J Korean Assoc Oral Maxillofac Surg 2019
Table 1. Gender and lesion size distributions of study subjects for assessment

\begin{tabular}{cr}
\hline Group & Value \\
\hline Sex & \\
Male & $14(70.0)$ \\
Female & $6(30.0)$ \\
Size $(\mathrm{cm})$ & \\
$>1-2$ & $5(25.0)$ \\
$>2-3$ & $9(45.0)$ \\
$>3-4$ & $6(30.0)$ \\
\hline
\end{tabular}

Values are presented as number (\%).

Vivekanand Sabanna Kattimani et al: Natural bioceramics: our experience with changing perspectives in the reconstruction of maxillofacial skeleton. J Korean Assoc Oral Maxillofac Surg 2019

Table 2. Radiological evaluation surgical site outlines versus elapsed time postsurgery $(\mathrm{n}=20)$

\begin{tabular}{ccccc}
\hline $\begin{array}{c}\text { Elapsed } \\
\text { time } \\
(\mathrm{wk})\end{array}$ & Unchanged & $\begin{array}{c}\text { Slightly } \\
\text { changed }\end{array}$ & $\begin{array}{c}\text { Partially } \\
\text { reduced }\end{array}$ & Absent \\
\hline 1 & $20(100)$ & 0 & 0 & 0 \\
4 & 0 & $12(60.0)$ & $8(40.0)$ & 0 \\
8 & 0 & 0 & $15(75.0)$ & $5(25.0)$ \\
12 & 0 & 0 & $1(5.0)$ & $19(95.0)$ \\
24 & 0 & 0 & 0 & $20(100)$ \\
\hline
\end{tabular}

Values are presented as number (\%).

Vivekanand Sabanna Kattimani et al: Natural bioceramics: our experience with changing perspectives in the reconstruction of maxillofacial skeleton. J Korean Assoc Oral Maxillofac Surg 2019
Table 3. Comparisons of radiological evaluation surgical site outlines over time intervals by Wilcoxon matched pairs test

\begin{tabular}{cccccccc}
\hline $\begin{array}{c}\text { Elapsed } \\
\text { time } \\
\text { (wk) }\end{array}$ & Mean & SD & $\begin{array}{c}\text { Me- } \\
\text { dian }\end{array}$ & $\begin{array}{c}\text { Mean } \\
\text { diff. }\end{array}$ & $\begin{array}{c}\% \text { of } \\
\text { change }\end{array}$ & Z-value & $P$-value \\
\hline 1 & 0.00 & 0.00 & 0.00 & & & & \\
4 & 1.40 & 0.50 & 1.00 & -1.40 & 100.00 & 3.9199 & $0.0001^{*}$ \\
4 & 1.40 & 0.50 & 1.00 & & & & \\
8 & 3.25 & 0.44 & 3.00 & -1.85 & -132.14 & 3.9199 & $0.0001^{*}$ \\
8 & 3.25 & 0.44 & 3.00 & & & & \\
12 & 3.95 & 0.22 & 4.00 & -0.70 & -21.54 & 3.2958 & $0.0010^{*}$ \\
12 & 3.95 & 0.22 & 4.00 & & & & \\
24 & 4.00 & 0.00 & 4.00 & -0.05 & -1.27 & 3.9199 & $0.0001^{*}$ \\
\hline
\end{tabular}

(SD: standard deviation, diff.: difference)

*Statistically significant difference $(P<0.05)$.

Vivekanand Sabanna Kattimani et al: Natural bioceramics: our experience with changing perspectives in the reconstruction of maxillofacial skeleton. J Korean Assoc Oral Maxillofac Surg 2019

Table 4. Radiological evaluation of bone formation with time postsurgery $(n=20)$

\begin{tabular}{ccccc}
\hline $\begin{array}{c}\text { Elapsed } \\
\text { time } \\
(\text { wk })\end{array}$ & Unchanged & $\begin{array}{c}\text { Ground glass } \\
\text { appearance }\end{array}$ & Spicular & Trabecular \\
\hline 1 & $20(100)$ & 0 & 0 & 0 \\
4 & 0 & $2(10.0)$ & $18(90.0)$ & 0 \\
8 & 0 & 0 & $18(90.0)$ & $2(10.0)$ \\
12 & 0 & 0 & $2(10.0)$ & $18(90.0)$ \\
24 & 0 & 0 & $1(5.0)$ & $19(95.0)$ \\
\hline
\end{tabular}

Values are presented as number (\%).

Vivekanand Sabanna Kattimani et al: Natural bioceramics: our experience with changing perspectives in the reconstruction of maxillofacial skeleton. J Korean Assoc Oral Maxillofac Surg 2019 
Table 5. Comparisons of radiological evaluation of bone formation over time intervals by Wilcoxon matched pairs test

\begin{tabular}{cccccccc}
\hline $\begin{array}{c}\text { Elapsed } \\
\text { time } \\
(\mathrm{wk})\end{array}$ & Mean & SD & $\begin{array}{c}\text { Me- } \\
\text { dian }\end{array}$ & $\begin{array}{c}\text { Mean } \\
\text { diff. }\end{array}$ & $\begin{array}{c}\% \text { of } \\
\text { change }\end{array}$ & Z-value & $P$-value \\
\hline 1 & 0.00 & 0.00 & 0.00 & & & & \\
4 & 1.90 & 0.31 & 2.00 & -1.90 & 100.00 & 3.9199 & $0.0001^{*}$ \\
4 & 1.90 & 0.31 & 2.00 & & & & \\
8 & 3.10 & 0.31 & 3.00 & -1.20 & -63.16 & 3.9199 & $0.0001^{*}$ \\
8 & 3.10 & 0.31 & 3.00 & & & & \\
12 & 3.90 & 0.31 & 4.00 & -0.80 & -25.81 & 3.5162 & $0.0004^{*}$ \\
12 & 3.90 & 0.31 & 4.00 & & & & \\
24 & 3.95 & 0.22 & 4.00 & -0.05 & -1.28 & 3.9199 & $0.0001^{*}$ \\
\hline
\end{tabular}

(SD: standard deviation, diff.: difference)

*Statistically significant difference $(P<0.05)$.

Vivekanand Sabanna Kattimani et al: Natural bioceramics: our experience with changing perspectives in the reconstruction of maxillofacial skeleton. J Korean Assoc Oral Maxillofac Surg 2019

complete radicular healing was observed in $80 \%$ of cases by the end of the eighth week and almost all subjects (90\%) exhibited complete healing by the end of the twelfth week.(Table 6 )

\section{Discussion}

Bioceramics is an advancing front in the reconstruction of defects of the maxillofacial skeleton. Various biomaterials have been evaluated as bone substitutes ${ }^{1-3}$. The volume needed for the reconstruction of maxillofacial defects without causing secondary morbidity in a patient with an autogenous graft is difficult to acquire, so alternative bone substitute materials have been investigated for a long time ${ }^{1}$. The cost involved for synthetic materials and autogenous grafting is not affordable to the common man, so the search for an economical ideal substitute material is ongoing ${ }^{4}$. In the recent past, eggshell derivatives have been assessed as scaffold materials ${ }^{11-14}$. More recently, an eggshell-derived bone graft substitute material was introduced and studied as a synthetic bone graft substitute in a few animal ${ }^{15-17}$ and human clinical studies $^{5,6,12}$.

Eggshell has been used for various medical formulations ${ }^{7,8}$. The eggshell contains calcium precursors, typically calcium carbonate $(94 \%)$, calcium phosphate $(1 \%)$, organic matter $(4 \%)$, and magnesium carbonate $(1 \%)^{7,8}$. The weight percentages of the constituents are $\mathrm{Ca}(35.11 \%), \mathrm{P}(3.66 \%), \mathrm{C}$ (15.28\%), O (44.59\%), and $\mathrm{Mg}(1.37 \%)^{7}$. Every day, a million tons of eggshells are produced as biological waste around the world ${ }^{7}$. Various production technologies for the synthesis of HA from different raw materials have been tried with varying success. Important issues in the industrial process are the minimization of waste and ability to recycle waste materials into useful products ${ }^{7}$. The synthesis of HA from chicken egg-
Table 6. Radiological evaluation of bone healing with elapsed time postsurgery $(n=20)$

\begin{tabular}{ccc}
\hline Elapsed time (wk) & Incomplete & Complete \\
\hline 1 & $20(100)$ & 0 \\
4 & $18(90.0)$ & $2(10.0)$ \\
8 & $4(20.0)$ & $16(80.0)$ \\
12 & $2(10.0)$ & $18(90.0)$ \\
24 & 0 & $20(100)$ \\
\hline
\end{tabular}

Values are presented as number (\%).

Vivekanand Sabanna Kattimani et al: Natural bioceramics: our experience with changing perspectives in the reconstruction of maxillofacial skeleton. J Korean Assoc Oral Maxillofac Surg 2019

shell has been tried in the laboratory with different methods like hydrothermal, wet precipitation, sol-gel, and microwave irradiation processes ${ }^{7}$. The nanotechnology-assisted production of HA as a bone substitute is a dream come true for the surgeons working on artificial bone regeneration ${ }^{15-17}$. The advantage of HA is its great compositional similarities to the main constituent of human bone and its ability to process osteoprogenitor cells ${ }^{1,3}$. The complex yet highly effective intracellular signaling of osteogenesis will be triggered by the presence of soluble calcium and inorganic phosphates ${ }^{1,3}$.

The ideal bone substitute should be biocompatible, osteoinductive or at least osteo-conductive, and have satisfactory mechanical properties ${ }^{11}$. This bone substitute should be available in unlimited quantities with low cost ${ }^{11}$. To accomplish these goals, the use of synthetic bone substitutes has been increasingly considered ${ }^{11}$. Eggshell powder has shown promising results in preliminary animal studies ${ }^{11-14}$. The experiments with animals in four groups have shown satisfactory healing, but osteo-induction has not been observed ${ }^{11,12}$. Eggshell powder has demonstrated no toxicity in animal experiments ${ }^{11-14}$. This natural product has already been used as a mineral additive in a chewing gum without adverse reaction; it is inexpensive and can be found in an unlimited quantity ${ }^{11}$. Recently, the eggshell powder has been processed and converted into HA, which has been evaluated in different animal models with promising results ${ }^{15-17}$. EHA has been produced by our associates using a domestic microwave process and this environmentally friendly conversion technique of conversion has been patented ${ }^{7}$. The preliminary studies have shown promising results in a small group of patients $\mathrm{s}^{5,6}$.

In the present study, the surgical site outline is the outline of the surgical defect after cystic enucleation, which will change with time because of creeping substitution from the periphery and will merge with the material margin in the due course of time ${ }^{9,10}$. The surgical site outlines were categorized as unchanged (altered less than 25\%), slightly changed (more than $25 \%$ and less than $75 \%$ ), partially reduced (more than 
$75 \%$ ), or absent (inability to differentiate between graft material outline and surgical site outline $)^{9,10}$. In the present study, the surgical site outline was slightly changed in 12 patients and partially reduced in eight patients by the fourth week and the eighth week, respectively. The surgical site margin visibility was further reduced to partially by the eighth week in 15 patients and was completely absent in five patients, indicating the material diffusion into the adjacent bone or the creeping of bony substitute from the periphery; this is similar to previous studies published in the literature ${ }^{5,6}$. The transition from the slightly reduced category to the partially reduced category was seen in seven patients over an interval of four weeks (i.e., from the fourth week to the eighth week).(Tables 2, 3) HA derived from eggshells showed more new bone formation in the early stages than did the scaffolds made from commercially available powders in animal studies ${ }^{16,17}$, and this is also evident in our radiographic observations of bone formation. Another animal study compared EHA and synthetic HA (SHA) using a rat model, which showed significantly higher bone regeneration in the EHA group than the unfilled control at eight weeks ${ }^{17}$. These results support our study findings. The merging of the material margin and the bony margin is indicative of new bone formation, as a creeping bone substitute and margins were invisible by the twelfth week.(Tables 2 , 3)

The formation of bone and the blending of the bone margin with the material margin were well correlated with the density of bone formation in the previous study ${ }^{6}$, which supports our study observations. The margin blend with commercially available materials like bovine and SHA in the previous studies are similar to those of the present study, indicating that EHA is equally efficient in the enhancement of bone regeneration $^{10}$. The rapid bone regeneration associated with grafting could be explained by the ability of the HA to enhance the selective adsorption of attachment proteins and growth factors that stimulate osteoblast adhesion and bone deposition ${ }^{18}$. Another mechanism by which the graft could enhance bone formation is through ion release, as supported by the work of Matsuoka and associates ${ }^{19}$.

The bone formation characteristics are the radiological appearance of the surgical site as changed, ground glass, spiculed, or trabecular ${ }^{9,10}$. These radiological appearances depend on the visible characteristics of radiographs in terms of radio-opacity in comparison with the adjacent bone and trabecular pattern. The study showed $90 \%$ specular appearance at the eighth week postoperatively, indicating the new bone formation. The initial radio-opacity of the graft material was increased within four weeks, which is indicative of the osteoconductive nature of the material.(Table 4) The study showed a trabecular pattern in 18 patients by the twelfth week, which indicated complete bone formation in $90 \%$ of the patients.(Table 4) The bone formation was significant during the time intervals from the fourth to the eighth week and from the eighth to the twelfth week.(Table 5) Kim et al. ${ }^{16}$ showed that the bone formation around the HA scaffolds fabricated from eggshells was more active than that of the control groups in rabbits. Similarly, Lee et al.'s animal study ${ }^{17}$ showed very similar results by the end of eight weeks, with $41.99 \% \pm 8.44 \%$ of new bone formation in SHA, a significantly higher amount, when compared with the control. Small-sized particles have a larger surface area than largesized particles with the same weight. Therefore, small-sized particles may be more easily removed from the body ${ }^{17}$, as our $\mathrm{HA}$ is in the form of nanoparticles, which might be the reason for early bone regeneration. A microwave process with low temperature was used to prepare the HA in our study ${ }^{7}$. The crystallinity of the EHA was higher than that of the SHA used in animal studies in the published literature ${ }^{17}$. Highly crystalline HA substrate supports higher osteogenic cell proliferation than low-crystalline HA substrates ${ }^{17}$. Most defect areas were filled with regenerated bone in the EHA group, and the remaining EHA particles were incorporated into the regenerated bone at eight weeks after the operation ${ }^{17}$. This might be the reason for the complete trabecular patterns of radiographs in our study. The trabecular pattern was similar with the adjacent normal bone. Similar findings have been reported in an animal study by Kim et al. ${ }^{16}$. The levels of new bone formation, lamellation, and maturation were greater in an EHA group compared to a synthetic group ${ }^{16}$. In addition to these obvious advantages of the enhancement of bone regeneration $^{5,6}$, the results of these studies suggest that EHA have excellent bone formation ability ${ }^{16,17}$. EHA was associated with a significantly higher bone formation in this study also.

The bone densities of the grafted sites were examined using Digora (Soredex, Helsinki, Finland). Digital intraoral periapical radiographs acquired at 4, 8, 12, and 24 weeks postsurgery were tabulated for changes in density with reference to the initial densities immediately after grafting, which were considered the baseline reference densities. The changes in density were correlated with bone formation characteristics at relevant time intervals. These changes in density were due to new bone formation and graft material resorption ${ }^{9,10}$. The marked density changes that were observed, indicating bone formation, are similar to results in the published literature ${ }^{5,6}$. 
The trabecular pattern of bone formation had the highest density attained at 12 weeks of follow-up; these findings are supported by our previous studies ${ }^{5,6,10}$ and other published literature using HA-modified bioglass ${ }^{18}$.

Calcium-deficient HA with surface microstructures is more favorable as a bone substitute compared to stoichiometric HA, because of its faster biodegradability and rapid formation of a surface apatite layer, enabling rapid bone-bonding microstructures ${ }^{15}$. Our EHA is calcium deficient, which might be the reason for the enhancement of bone regeneration. Park et al. ${ }^{15}$ showed that surface characteristics may contribute to the increased osteo-conduction of hydrothermally treated eggshells in the healing of rat calvarial defects, thus, the HA derived from chicken eggshell is an osteoconductive material for the enhancement of early bone regeneration ${ }^{15-17}$. Recent studies provided proof of concept for the clinical application of "smart" biomaterials, because the HA's osteo-inductive nature displayed superior biological performance through the modulation of cell behavior ${ }^{20-22}$. EHA-enhanced bone formation, and healing was complete by the end of 12 weeks.(Table 6) This enhancement might be because of the calcium-deficient nature of EHA. The calcium-deficient characteristics of the surface HA layer in the new bone graft substitute (N-HA) derived from hen eggshell in Park et al.'s study ${ }^{23}$ showed promotion of new bone formation by increasing protein adsorption and supplying sufficient $\mathrm{Ca}$, which enhanced the reprecipitation and formation of calcium phosphate layers on its surface and subsequently influenced osteoblast responses. Even the histomorphometric observations revealed new bone formation and new bone islands with N-HA derived from hen eggshell prepared through hydrothermal processing, which is osteoconductive in nature ${ }^{23}$. Our radiographic bone formation characteristics might be enhanced due to these abovementioned facts at early stages within 8 to 12 weeks. The study samples were divided into three groups depending on the size of the lesions: $>10-20 \mathrm{~mm},>20-30 \mathrm{~mm}$, and $>30-40$ mm.(Table 1) However, the healing of bone was complete in all of the patients irrespective of the sizes of their lesions. The literature showed that tunnel lesions will heal with scarring or fibrous connective tissue if grafting is not attempted ${ }^{24,25}$. The published literature showed the necessity of grafting for early bone formation and enhancement, which supports our findings ${ }^{23-25}$. The majority of our cases were tunnel lesions; $45 \%$ of the patients had lesions $>20-30 \mathrm{~mm}$ in size, and $30 \%$ had lesions $>30-40 \mathrm{~mm}$ in size. In all cases, the healing was uneventful; $80 \%$ of patients showed complete bone healing by the eighth week, and $90 \%$ by the twelfth week. Within the group, the study didn't show any significant differences in the healing among all of the lesions. Regenerative therapy is essential for the enhancement of bone healing so that grafting prevents scar tissue formation ${ }^{24,25}$. The published literature supports the notion of grafting/regenerative techniques for early bone healing ${ }^{26-29}$. However, none of the published studies used EHA. They used different grafting materials, including two studies that had control groups with Chitra granules $^{27}$ and anorganic bovine bone ${ }^{28}$, and one study that used a freeze-dried bone allograft without a control group ${ }^{29}$. All of these studies suggested that grafting or regenerative techniques enhance bone formation compared to control groups $^{28,29}$.

In our study, wound dehiscence was seen in one case, which was attributed to improper surgical planning and an existing sinus within the flap. This case has been excluded from the evaluation due to seepage of graft material through the defect. None of the animal studies in the published literature showed any undue reactions to the EHA bone graft substitute $^{15-17,23}$. Eggshells are by products of food waste, so recycling them as a raw material for bone graft material production; is not only economical but also environment friendly because of ready availability of eggshell waste ${ }^{17,23}$. EHA is cost-effective and production is environment friendly with no disease transfer risks ${ }^{5,6}$. This economical graft material can be used as raw material for the production of various shapes and sizes of grafts in various biomedical applications including three-dimensional construction ${ }^{30}$.

\section{Conclusion}

Our study showed early bone regeneration by the twelfth week and EHA-enhanced bone formation. The grafting prevented dead space and undue complications associated with a large hematoma in the surgical defect. The EHA acts as a space filler and osteoconductive economic graft material. It is a versatile biocompatible graft substitute that does not cause any chronic inflammatory, allergic, or toxic reactions. Thus, bioceramics derived from natural calcium precursors like chicken eggshell (natural bio-waste) will play an important role in the reduction of the cost involved with grafting because of the abundant availability of this raw material.

\section{ORCID}

Vivekanand Sabanna Kattimani, https://orcid.org/00000002-9812-7207 
Krishna Prasad Lingamaneni, https://orcid.org/0000-00020098-2900

\section{Authors' Contributions}

V.S.K. performed study, participated in data collection and wrote the manuscript. V.S.K. and K.P.L. designed and analyzed the study. All authors read and approved the final manuscript.

\section{Acknowledgements}

We thank the Head and colleagues of the Department of Conservative Dentistry and Endodontics and Radiology for their support during execution of this study protocol; Dr. E. K. Girija, Department of Physics, Periyar University Salem, and the Department of Science and Technology Government of India for their funding for the synthesis and scaling up eggshell-derived HA as a collaborative Waste Management Technology project. Also, thanks to Hon. Vice-Chancellor and Registrar of Dr. NTR University of Health Sciences, Vijayawada for providing an opportunity to carry out this research work as a part of a Ph.D. study.

This study was supported by Department of Science and Technology, Government of India (grant no. DST/TSG/ $\mathrm{WM} / 2015 / 576 / \mathrm{G})$.

\section{Ethics Approval and Consent to Participate}

The study protocol was approved by the Institutional Ethics Committee of Sibar Institute of Dental Sciences (IEC 16/09/2014) and prospectively registered with the Clinical Trial Registry of India - CTRI/2014/12/005340.

\section{Conflict of Interest}

No potential conflict of interest relevant to this article was reported.

\section{References}

1. Thrivikraman G, Athirasala A, Twohig C, Boda SK, Bertassoni LE. Biomaterials for craniofacial bone regeneration. Dent Clin North Am 2017;61:835-56.

2. Gilbert Triplett R, Budinskaya O. New frontiers in biomaterials. Oral Maxillofac Surg Clin North Am 2017;29:105-15.

3. Adamopoulos O, Papadopoulos T. Nanostructured bioceramics for maxillofacial applications. J Mater Sci Mater Med 2007;18:1587-
97.

4. Kattimani VS, Chakravarthi PS, Prasad LK. Biograft block hydroxyapatite: a ray of hope in the reconstruction of maxillofacial defects. J Craniofac Surg 2016;27:247-52.

5. Kattimani VS, Chakravarthi PS, Kanumuru NR, Subbarao VV, Sidharthan A, Kumar TS, et al. Eggshell derived hydroxyapatite as bone graft substitute in the healing of maxillary cystic bone defects: a preliminary report. J Int Oral Health 2014;6:15-9.

6. Kattimani V, Lingamaneni KP, Chakravarthi PS, Kumar TS, Siddharthan A. Eggshell-derived hydroxyapatite: a new era in bone regeneration. J Craniofac Surg 2016;27:112-7.

7. Siva Rama Krishna D, Siddharthan A, Seshadri SK, Sampath Kumar TS. A novel route for synthesis of nanocrystalline hydroxyapatite from eggshell waste. J Mater Sci Mater Med 2007;18:1735-43.

8. Suresh Kumar G, Girija EK. Flower-like hydroxyapatite nanostructure obtained from eggshell: a candidate for biomedical applications. Ceram Int 2013;39:8293-9.

9. Kattimani VS, Bajantai NV, Sriram SK, Sriram RR, Rao VK, Desai PD. Observer strategy and radiographic classification of healing after grafting of cystic defects in maxilla: a radiological appraisal. J Contemp Dent Pract 2013;14:227-32.

10. Kattimani VS, Chakravarthi SP, Neelima Devi KN, Sridhar MS, Prasad LK. Comparative evaluation of bovine derived hydroxyapatite and synthetic hydroxyapatite graft in bone regeneration of human maxillary cystic defects: a clinico-radiological study. Indian J Dent Res 2014;25:594-601.

11. Dupoirieux L, Pourquier D, Souyris F. Powdered eggshell: a pilot study on a new bone substitute for use in maxillofacial surgery. J Craniomaxillofac Surg 1995;23:187-94.

12. Baliga M, Davies P, Dupoirieux L. [La poudre de coquille d'oeuf dans le comblement des cavitescystiques des maxillaires]. Rev Stomatol Chir Maxillofac 1998;99 Suppl 1:86-8. French.

13. Dupoirieux L. Ostrich eggshell as a bone substitute: a preliminary report of its biological behaviour in animals--a possibility in facial reconstructive surgery. Br J Oral Maxillofac Surg 1999;37:467-71.

14. Dupoirieux L, Neves M, Pourquier D. Comparison of pericranium and eggshell as space fillers used in combination with guided bone regeneration: an experimental study. J Oral Maxillofac Surg 2000;58:40-6; discussion 47-8.

15. Park JW, Bae SR, Suh JY, Lee DH, Kim SH, Kim H, et al. Evaluation of bone healing with eggshell-derived bone graft substitutes in rat calvaria: a pilot study. J Biomed Mater Res A 2008;87:203-14.

16. Kim SH, Kim W, Cho JH, Oh NS, Lee MH, Lee SJ. Comparison of bone formation in rabbits using hydroxyapatite and $\beta$-tricalcium phosphate scaffolds fabricated from egg shells. Adv Mater Res 2008;47-50:999-1002.

17. Lee SW, Kim SG, Balázsi C, Chae WS, Lee HO. Comparative study of hydroxyapatite from eggshells and synthetic hydroxyapatite for bone regeneration. Oral Surg Oral Med Oral Pathol Oral Radiol 2012;113:348-55.

18. El-Ghannam A, Amin H, Nasr T, Shama A. Enhancement of bone regeneration and graft material resorption using surface-modified bioactive glass in cortical and human maxillary cystic bone defects. Int J Oral Maxillofac Implants 2004;19:184-91.

19. Matsuoka H, Akiyama H, Okada Y, Ito H, Shigeno C, Konishi J, et al. In vitro analysis of the stimulation of bone formation by highly bioactive apatite- and wollastonite-containing glass-ceramic: released calcium ions promote osteogenic differentiation in osteoblastic ROS17/2.8 cells. J Biomed Mater Res 1999;47:176-88.

20. Yuan H, Fernandes H, Habibovic P, de Boer J, Barradas AM, de Ruiter A, et al. Osteoinductive ceramics as a synthetic alternative to autologous bone grafting. Proc Natl Acad Sci U S A 2010; 107:13614-9.

21. Ripamonti U. Osteoinduction in porous hydroxyapatite implanted in heterotopic sites of different animal models. Biomaterials 1996;17:31-5.

22. Gosain AK, Song L, Riordan P, Amarante MT, Nagy PG, Wilson 
CR, et al. A 1-year study of osteoinduction in hydroxyapatitederived biomaterials in an adult sheep model: part I. Plast Reconstr Surg 2002;109:619-30.

23. Park JW, Jang JH, Bae SR, An CH, Suh JY. Bone formation with various bone graft substitutes in critical-sized rat calvarial defect. Clin Oral Implants Res 2009;20:372-8.

24. von Arx T, Alsaeed M. The use of regenerative techniques in apical surgery: a literature review. Saudi Dent J 2011;23:113-27.

25. Deng Y, Zhu X, Yang J, Jiang H, Yan P. The effect of regeneration techniques on periapical surgery with different protocols for different lesion types: a meta-analysis. J Oral Maxillofac Surg 2016;74:239-46.

26. Alnemer NA, Alquthami H, Alotaibi L. The use of bone graft in the treatment of periapical lesion. Saudi Endod J 2017;7:115-8.
27. Sreedevi P, Varghese N, Varugheese JM. Prognosis of periapical surgery using bonegrafts: a clinical study. J Conserv Dent 2011;14:68-72.

28. Taschieri S, Del Fabbro M, Testori T, Saita M, Weinstein R. Efficacy of guided tissue regeneration in the management of throughand-through lesions following surgical endodontics: a preliminary study. Int J Periodontics Restorative Dent 2008;28:265-71.

29. Saad AY, Abdellatief EM. Healing assessment of osseous defects of periapical lesions associated with failed endodontically treated teeth with use of freeze-dried bone allograft. Oral Surg Oral Med Oral Pathol 1991;71:612-7.

30. Gaviria L, Pearson JJ, Montelongo SA, Guda T, Ong JL. Threedimensional printing for craniomaxillofacial regeneration. J Korean Assoc Oral Maxillofac Surg 2017;43:288-98. 